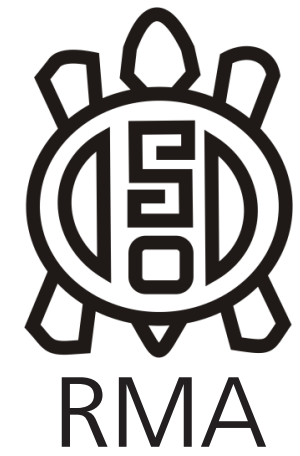

Dossier

\title{
Primeros avances en la caracterización de la tecnología lítica en el Holoceno final del norte de San Luis
}

\author{
First advances in the characterization of the lithic technology in \\ the final Holocene from the north of San Luis
}

Mariana Ávila Navas*

*Escuela de Arqueología- Universidad Nacional de Catamarca. E-mail: marianrachel6@gmail.com

\begin{abstract}
Resumen
En este trabajo se abordará el análisis técnico morfológico de una muestra lítica obtenida en el Chimborazo 1, ubicado en un sector semi-árido del norte de la provincia de San Luis, Argentina. Se trata de un sitio a cielo abierto, donde se visualizan hornillos y abundante material lítico disperso en antiguas terrazas aluviales del arroyo Los Hornos. Actualmente se encuentra sometido a un proceso de erosión que moviliza los materiales superficiales y destruye hornillos gradualmente. En ese marco, las recolecciones superficiales permitieron recuperar elementos para analizar desde la propuesta de la Organización de la Tecnología. El mismo estuvo enfocado en brindar un primer acercamiento sobre la gestión tecnológica que se estaba desarrollando en el sitio, como así también las instancias, técnicas de talla y materias primas utilizadas. Este primer análisis indica que se llevaba a cabo un proceso de talla que involucraría hasta las etapas intermedias, con la presencia tanto de desechos y núcleos como instrumentos realizados con diferentes materias primas (cuarcita, cuarzo y calcedonia). Dichos instrumentos estarían relacionados con posibles tareas de corte y raspado.
\end{abstract}

Palabras clave: Tecnología lítica; Holoceno final; Norte de San Luis; Gestión tecnológica; Primeras evidencias.

\begin{abstract}
In this paper, will be approach the morphological technical analysis of a lithic sample obtained in Chimborazo 1, located in a semi-arid sector in the north of the province of San Luis, Argentina. It is an open-air site, where stoves and abundant lithic material scattered on ancient alluvial terraces of Los Hornos stream are displayed. It is currently undergoing an erosion process that mobilizes surface materials and gradually destroys stoves. Within that framework, surface collections allowed recovering elements to analyze from the proposal of the Technology Organization. It was focused on providing a first approach to the technological management that was being developed on the site, as well as the instances, size techniques and raw materials used. This first analysis indicates that a process of continuous size was carried out that would involve up to the intermediate stages, with the presence of debitage, cores and instruments made with different raw materials (quartzite, quartz and chalcedony). These instruments would be related to possible cutting and scraping tasks.
\end{abstract}

Keywords: Lithic technology; Final holocene; North of San Luis; Management tecnology; First evidences.

\section{Introducción}

El sitio arqueológico EL Chimborazo 1 (ECH1 en adelante) se encuentra ubicado en el sector semi-árido del norte de la provincia de San Luis (Figura 1). Dispuesto en el ecotono entre las provincias fitogeográficas del Monte y Chaco Seco (Cabrera, 1976). Se trata de un sitio a cielo abierto, identificado sobre un curso efímero discontinuo (Bull 1997) activo durante la época estival. Debido a su localización, y su composición de rocas sedimentarias (lutitas, areniscas y conglomerados), presenta un importante deterioro, que se manifiesta en forma de abanicos de canal y cárcavas (Candia Halupczok, 2015). Los primeros trabajos en el sitio ECH1 tuvieron como objetivos conocer la dinámica geomorfológica y geológica del mismo, para entender el funcionamiento ambiental de la región durante la ocupación humana (Ojeda et al., 2018; Heider et al., 2018). En el marco de este último trabajo multidisciplinario se obtuvieron dos fechados radiocarbónicos, ambos del Holoceno final. El más antiguo, (Beta 470121, $1780 \pm 30 \mathrm{cal}$. AP), corresponde a la datación realizada en un paleosuelo. El segundo, (Beta $470120,540 \pm 30 \mathrm{cal}$. AP) remite al análisis de carbones del fondo de un hornillo ubicado sobre una barranca. En ese contexto de divergencia estratigráfica se están realizando estudios tendientes a proponer sincronías o diacronías entre ocupaciones. En ese marco, todos los materiales analizados provienen de una recolección 
superficial realizada en el ECH1, correspondiente actualmente a una terraza de inundación. La misma se ve afectada por el desborde del cauce durante la época estival. Los materiales expuestos corresponderían a la ocupación más reciente en el sitio, propia del momento geológico local conocido como Fm. Los Algarrobitos, ca. 500 AP (Dr. Guillermo Heider com. pers.). Con anterioridad a los estudios mencionados no se han realizado abordajes sistemáticos en el norte de San Luis. Sin embargo, existen indagaciones a escala regional, realizadas por Outes y Vignati en la primera parte del siglo XX. En sus trabajos, los autores presentan el registro descriptivo de Hornillos o Tinajas de tierra cocida en distintos parajes del norte puntano (Balde de Azcurra, La Botija, Pampa de las Salinas, entre otros), en asociación a instrumentos líticos correspondientes a puntas de proyectil lanceoladas, raederas y raspadores, confeccionadas principalmente en cuarzo y cuarcitas (Outes, 1926; Vignati, 1938).

Este trabajo tiene como objetivo determinar qué técnicas e instancias de manufactura se estaban realizando en el ECH1. Adicionalmente, nos interesa generar un primer acercamiento a las estrategias y la dinámica tecnológica lítica, indagando sobre las materias primas explotadas, como así también, las características que pudieron contemplarse al momento de la elección de los materiales para la talla. Para ello, se analizó una muestra bajo los criterios técnico tipológico y morfológico funcional formulados por Aschero (1975; 1983), Aschero

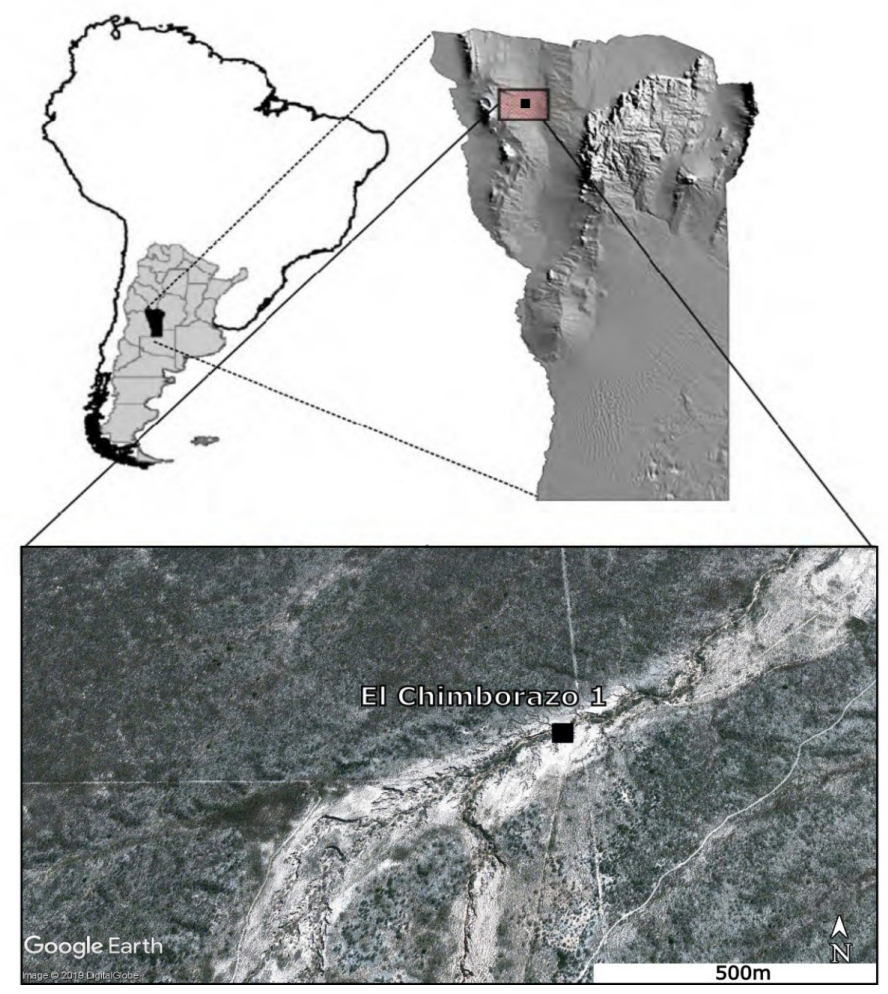

Figura 1: Mapa de ubicación del área en estudio.

Figure 1: Location map of the site under study. y Hoscman (2004) y los postulados de la organización tecnológica propuestos por Nelson (1991). La muestra analizada en este trabajo sumó un total de 403 piezas recolectadas mediante un muestreo probabilístico estratificado (Renfrew y Bahn, 1998). Las cuadrículas de recolección, de $1 \times 1$ metro, fueron planteadas en una terraza de inundación afectada por numerosas cárcavas actualmente activas. Cada cuadricula (denominadas de $\mathrm{A}$ a $\mathrm{C}$ ) fue planteada en diferentes puntos de una cárcava lateral, separadas por 5 metros en su ángulo noreste (Figura 2). En este sentido, su ubicación estuvo relacionada con la necesidad de recoger materiales que suponíamos afectados de modo diferencial por el proceso erosivo, pero, que a la vez formaran parte de un conjunto reconocible a simple vista a nivel superficial. En los sectores menos afectados (el más alejado de una profunda cárcava central) se recuperaron 79 elementos (unidad de recolección A). En el sector medio o unidad B, cuya pendiente comienza a agudizarse, la muestra ascendió a 279. Finalmente, en la unidad C, donde disminuye el declive se obtuvieron 45 piezas. Del total de la muestra, 180 fueron catalogados como ecofactos (23 correspondientes a la unidad A, 119 a la B y 38 a la C), cuyo análisis no será incluido en el presente trabajo.

\section{Resultados}

La muestra analizada tiene un total de 223 piezas, dividida en 205 desechos, 12 instrumentos y 6 núcleos. La totalidad de la misma está compuesta por un número reducido de materias primas: el $55,16 \%$ es cuarcita $(n=123)$, seguida por un $25,11 \%$ de cuarzo $(n=56)$ y un $18,38 \%$ de calcedonia $(n=41)$ y $1,35 \%$ de materias primas no diferenciadas $(n=3)$.

\section{Cuadrícula A}

De esta unidad de muestreo se analizó un total de 22 piezas de los cuales 20 son desechos de talla (91\%) y 2 son núcleos (9\%). En el subconjunto de los desechos se hace presente un predominio de las lascas primarias (37\%), seguido por lascas angulares (31\%), de arista (13\%), planas (13\%) y secundarias (6\%) (Tabla 1). Respecto a los talones, el $67 \%(n=10)$ fueron identificados como lisos con corteza natural, un 13\% ( $n=$ 2) puntiformes y el $20 \%(n=3)$ restante fueron identificados como lisos (6\%), diedros (7\%) y filiformes (7\%). Se observó una marcada predominancia de desechos con presencia de restos de corteza (90\%). Un 60\% $(n=12)$ se encuentran fracturados con presencia de talón, el $20 \%(n=5)$ de los casos no se les ha podido diferenciar sus partes diagnósticas por lo que fueron catalogados como debris, un $15 \%(n=3)$ se presentan enteros y sólo un $5 \%$ $(n=1)$ se encuentran fracturadas sin presencia 


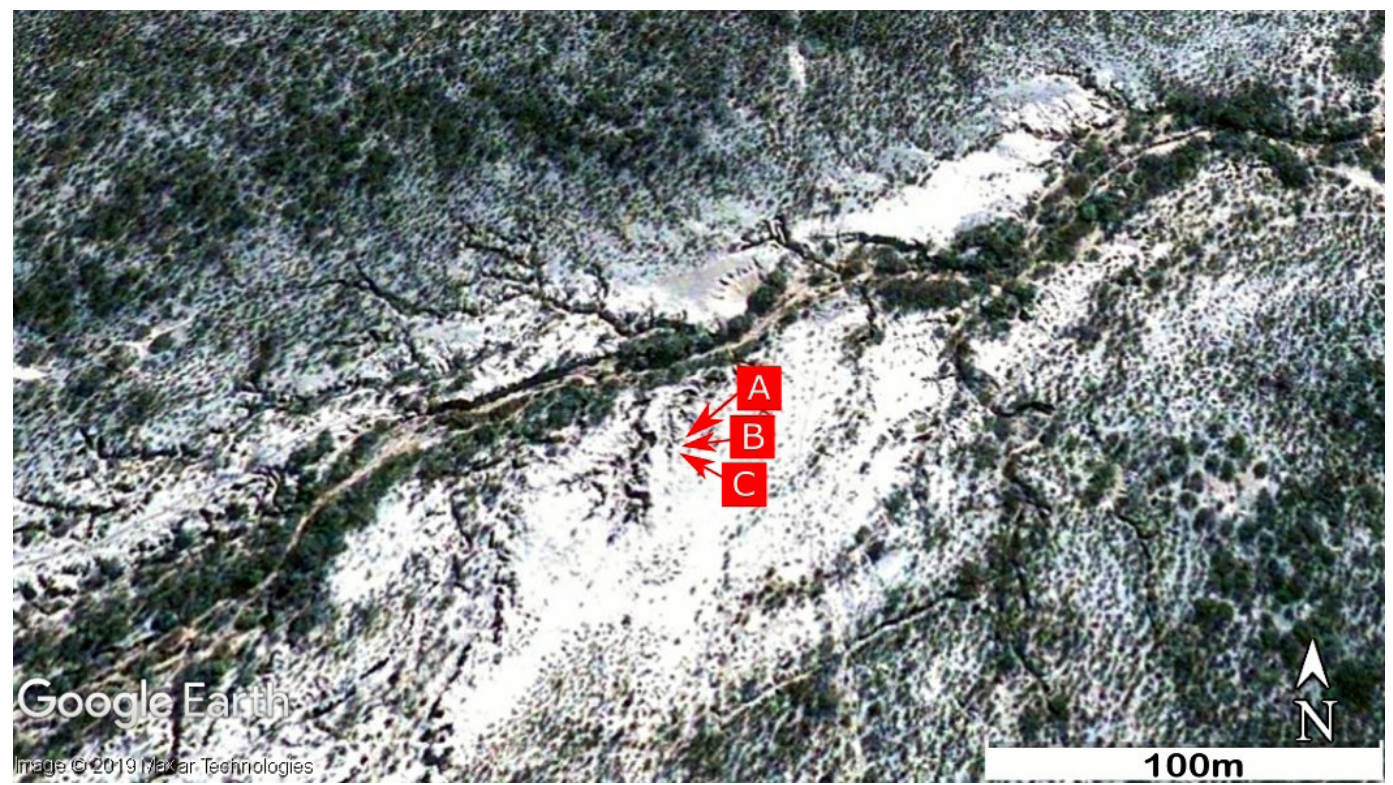

Figura 2: Ubicación de las cuadrículas de recolección.

Figure 2: Location of collection grids.

de talón. En cuanto a variables dimensionales de los desechos se observa una mayor representación de los desechos de tamaño 'mediano pequeño' $(n=10)$ grande $(n=5)$ y muy grande $(n=3)$. En cuanto a los Módulos de longitud/anchura, hay una mayor cantidad de tamaño mediano normal $(n=9)$ y corto ancho $(n=8)$ pero con representación de los demás en una mínima unidad. Respecto a los núcleos, se identificaron 2. El primero bifacial, de cuarcita con lascados bifaciales alternados cuya forma de contorno corresponde a pentagonal irregular sin ápice aguzado. El segundo realizado en cuarzo presenta lascados aislados con forma trapezoidal asimétrico. Este último posee un máximo de 9 extracciones, mientras que el bifacial presenta 20 .

\section{Cuadrícula $B$}

Esta cuadrícula presenta una mayor cantidad de piezas analizadas $(n=160)$. Se diferenciaron 148 desechos de talla $(92,5 \%), 8$ instrumentos $(5 \%)$ y 4 núcleos $(2,5 \%)$. En el primer conjunto, el 54\% $(n=80)$ de la muestra se encuentra fracturada con presencia de talón, un 23\% $(n=35)$ entero, $12 \%(n=17)$ no diferenciada (debris) y un $11 \%(n=16)$ fracturada sin talón. Respecto a los tipos de talones hay un claro predominio de los lisos-natural $(n=47)$ y los lisos $(n=42)$. Dentro de esta cuadrícula, se vieron más representadas las lascas angulares (33\%), lascas planas (13\%), lascas de arista simple (12\%) y primarias (8\%) entre otras (Tabla 1). Se pudo observar la presencia de corteza en un $91 \%$ de los elementos analizados, en distintas proporciones. Las dimensiones predominantes fueron mediano pequeño $(n=64)$ y pequeño $(n=51)$ para tamaño; en el caso de módulo de longitud-anchura, figuran en primer lugar los mediano normal $(n=52)$ y corto ancho $(n=33)$.

Los instrumentos identificados fueron 8, realizados en cuarcita $(n=4)$ y calcedonia $(n=4)$. Los grupos tipológicos representados fueron cortantes $(n=2)$, raspadores $(n=2)$, denticulados $(n=2)$, muesca $(n=1)$, cuchillo $(n=1)$ y punta de proyectil $(n=1)$. Se destaca en este conjunto es la presencia de dos instrumentos compuestos: el primero realizado en cuarcita contiene un cortante y una muesca, mientras que el segundo fue confeccionado en calcedonia y se trata de un núcleo bifacial que en uno de sus extremos presenta un raspador con filo frontal realizado con lascados simples de formatización marginal de forma paralelo corto regular. Estas tres últimas características son las que predominan en la confección de los demás instrumentos, que presentan además situación de los lascados directos, pero una forma primaria de los biseles variable con asimétrica unifacial $(n=5)$ y simétrico unifacial $(n=2)$. Un segundo raspador presenta características similares al compuesto mencionado. Sólo se presentó un caso de simetría bifacial cuya serie técnica también se destacaba al ser parcialmente extendida. Se trata de un denticulado con filo irregular oblicuo de calcedonia cuya forma base no se ha podido diferenciar. En cuanto a los instrumentos restantes sus formas bases las constituyen lascas secundarias y un núcleo bifacial. Las dimensiones más representativas del total de los mismos fueron tamaño grande $(n=4)$ con un módulo de longitud anchura mediano normal $(n=4)$. Como un caso aparte, la única punta de proyectil recuperada de esta cuadrícula presenta una forma del contorno correspondiente a una lanceolada con base acuminada convexilínea, realizada con retoque parcialmente extendido bifacial paralelo corto irregular de tamaño pequeño y un módulo mediano alargado. La misma fue confeccionada en una lasca de calcedonia. Respecto a los núcleos $(n=4)$, como se mencionó anteriormente, se identificó uno bifacial de calcedonia con lascados alternos de similares características con un contorno no tipificable. En los casos restantes, el tipo de núcleo no pudo ser identificado. Sin embargo, en dos ocasiones se pudieron diferenciar la forma del contorno en trapezoidal asimétrico y triangular irregular sin ápice aguzado, respectivamente. Estos dos últimos de cuarcita, mientras que el no tipificable es de cuarzo. Los números de planos de percusión van desde 6 a 12 
M. Ávila Navas | Revista del Museo de Antropología 13 (1): 267-272

DOI: http://doi.org/10.31048/1852.4826.v13.n1.23835

Tabla 1: Tipos de desechos de talla representados en la muestra.

Table 1: Types of debitage represented in the sample.

\begin{tabular}{c|r|r|r|r|r|r|r|r|r|}
\hline $\begin{array}{c}\text { Cuadrícula de } \\
\text { recolección }\end{array}$ & Primarias & Secundarias & $\begin{array}{c}\text { Dorso } \\
\text { Natural }\end{array}$ & Planas & $\begin{array}{c}\text { Arista } \\
\text { Simple }\end{array}$ & $\begin{array}{c}\text { Arista } \\
\text { Doble }\end{array}$ & Angulares & $\begin{array}{c}\text { Flanco de } \\
\text { Núcleo }\end{array}$ & $\begin{array}{c}\text { No } \\
\text { diferenciada }\end{array}$ \\
\hline A & 6 & & & 2 & 2 & & 5 & & \\
\hline B & 13 & 9 & 5 & 22 & 18 & 6 & 50 & 2 & 6 \\
\hline C & 4 & 4 & 9 & 3 & 3 & 1 & 11 & 7 & 7 \\
\hline$\%$ & 11,22 & 6,34 & 6,83 & 13,17 & 11,21 & 3,41 & 32,19 & 0,97 & 3,41 \\
\hline
\end{tabular}

aproximadamente.

\section{Cuadrícula C}

Está constituida en primera instancia por 41 piezas analizadas, de los cuales $37(90 \%)$ son desechos y $4(10 \%)$ son instrumentos. Dentro de los primeros, un $70 \%(n=26)$ presentaba fractura con presencia de talón, un $22 \%(n=8)$ se encontraba entero, el $7 \%(n=3)$ restante se lo identificó como fracturado sin talón y no identificado. Respecto a los talones se ha identificado predominantemente lisosnaturales $(n=16)$ lisos sin presencia de corteza $(n=6)$ y puntiformes $(n=6)$. Los tipos de desechos identificados muestran un predominio de las lascas angulares 31\%, seguida de las de dorso natural en un $25 \%$, viéndose representados otros tipos como las secundarias (14\%) y primarias (11\%), entre otras (Tabla 1). El $84 \%$ de los desechos presentó corteza. Los instrumentos encontrados en esta cuadrícula $(n=4)$ corresponden a una punta de proyectil, un cortante y dos cuchillos. Estos tres últimos, mostraron filos naturales y presencia de rastros complementarios con microrretoque marginal, simétrico unifacial. En el caso de los cuchillos de cuarcita, las formas bases estaban constituidas por lascas angulares. Mientras que el cortante fue realizado en una lasca primaria. La punta de proyectil, al igual que el cortante, se encuentra realizada en cuarzo cristalino. La misma posee una forma de contorno triangular realizada por retalla extendida cuya base corresponde a una concavilínea simple, de tamaño mediano pequeño y módulo mediano alargado. Presenta una fractura que le ocasionó la pérdida del ápice.

\section{Discusión y Consideraciones finales}

Las investigaciones arqueológicas en el ECH1 son parte de una tesis de Licenciatura en desarrollo, la cual se incluye en un proyecto mayor, orientado a estudiar a los pueblos originarios de las zonas áridas y semi-áridas de Las Travesías de San Luis. Para esta contribución, preliminar, nos centramos en presentar resultados tendientes a caracterizar la gestión tecnológica del sitio. En ese sentido, los desechos de talla muestran un predominio de las lascas de etapas intermedias e iniciales de manufactura. Presentándose en un $66 \%$ fracturado con presencia de talón. Los mismos fueron predominantemente, lisos- naturales (40\%) de forma contundente, relacionados generalmente a la utilización de técnicas de talla directa por percusión. Los tamaños fueron variados, sin embargo, se observó un predominio del mediano pequeño (55\%). Respecto al módulo de longitud-anchura el mediano normal representó el $48 \%$ de la muestra, seguido por corto ancho en un $22 \%$. Estos datos pueden dar cuenta de un proceso abarcativo de las primeras etapas de talla, correspondientes al descortezamiento de los núcleos y de las lascas llegando a etapas intermedias constituyendo artefactos con filos útiles y con mayor formatización.

Dentro de los instrumentos identificados $(n=12)$, las formas base más recurrentes para la confección de los mismos fueron lascas secundarias y angulares con presencia de corteza. El porcentaje de fractura en los mismos fue del $60 \%$. Los filos predominantes fueron los unifaciales, tanto asimétricos como simétricos, elaborados con lascados simples de formatización, siendo la forma de dichos lascados paralelo corto irregular $(n=5)$ y ultramarginal $(n=4)$ en su mayoría. Estos artefactos indicarían la realización de actividades variables, predominando las de corte y raspado (Figura 3). Ejemplo de esto es la presencia de instrumentos compuestos. Este tipo de instrumentos pueden considerarse versátiles al poseer varios filos funcionales aptos para distintas tareas, buscando la simplificación de varias herramientas y sus respectivas funciones en una sola (Nelson, 1991). La aparición de puntas de proyectil da indicios de posibles tareas de confección y mantenimiento de herramientas destinadas a la caza o defensa, sumando la presencia de una muesca, la cual podría ser utilizada en la fabricación de astiles (Winchkler, 2006). Teniendo en cuenta los aspectos mencionados, así como otros rasgos del registro arqueológico no presentados en este trabajo (i.e.hornillos, cerámica, fogones) se considera, a modo de propuesta inicial, que el sitio ECH1 fue una base residencial.

Las materias primas más representativas de la muestra total analizada fueron la cuarcita y el cuarzo. Estas son rocas consideradas relativamente aptas para la talla, pese a su dureza, sobre todo la primera que al ser de grano más fino facilita el desprendimiento de lascas según lo desee el tallador. La calcedonia, por su parte, 
Figura 3: Instrumentos identificados en $\mathrm{ECH} 1$.

Figure 3: Instruments identified in ECH1.

es la menos representada en la muestra, aunque esto no impidió que sea la segunda más utilizadas para la confección de instrumentos $(n=4)$. Pese a las inclusiones $u$ oquedades propias de esta roca que generaron una gran cantidad de desechos no identificados (debris) es considerada una buena materia prima para la talla. Los porcentajes que se registraron de cada una de las materias primas pueden deberse, entendemos, no solo a sus cualidades sino también a la accesibilidad de las mismas, siendo la cuarcita la más elegida.

Los núcleos identificados presentan todavía parte de la corteza por lo que puede considerarse que las fuentes se encontraban próximas al sitio (Franco, 1991). El tamaño de ninguno de los mismos superan los 9,5 cm de diámetro, una característica favorable para pensar en el transporte de éstos hasta ECH1.

Los análisis realizados permiten visibilizar, por el momento, con mayor claridad estrategias de índole expeditivas, considerando la presencia de filos naturales y de lascados simples de formatización en los instrumentos. Esto mostraría poca inversión de trabajo a la hora de la talla; primando las lascas angulares y secundarias como formas base; siendo las puntas de proyectil la excepción, ya que demandan una mayor inversión de tiempo y trabajo. Sin embargo, la presencia de núcleos bifaciales nos plantea la posibilidad de que en ECH1 se hayan llevado a cabo estrategias de índole conservadoras. Por lo que creemos necesario ampliar la muestra en un futuro cercano.

De esta manera, tampoco se puede afirmar que en el sitio no se haya realizado reactivación de biseles, otro potencial indicador de estrategias conservadoras, ya que la falta de evidencias puede no ser el reflejo de las acciones humanas sino de los aspectos tafonómicos del sitio. Con esto último, hacemos referencia principalmente a la erosión fluvial la cual pudo arrastrar la mayor parte del material lítico más pequeño y liviano.

A partir de estos primeros avances se proyectarán nuevos objetivos; ampliando la muestra con nuevo material lítico recolectado en el sitio por medio de transectas longitudinales. De esta forma se buscará comprender la organización de la tecnología en ECH1 y aquellas

variables que pudieron incidir en las estrategias y diseños; los cuales podrían estar vinculados a la búsqueda de los recursos vitales para la subsistencia en estas regiones de Travesías. Como así también, se indagará sobre aspectos de interacción y movilidad a partir de las evidencias disponibles sobre fuentes de aprovisionamiento. En futuros trabajos se incluirá también el análisis de los ecofactos, los que darían información concerniente a cómo ingresaban las materias primas a ECH1, los percutores que se pudieron haber utilizado para tallar $u$ otro tipo de actividades que se habrían realizado en el lugar.

Papagayos, San Luis, 16 de Marzo 2019.

\section{Agradecimientos}

Al gobierno de la provincia de San Luis y a los pueblos originarios. A la familia Atensio y a don Guillermo. A mis directores Guillermo Heider y Enrique Moreno por su apoyo. Finalmente, un agradecimiento especial a la Dra. Patricia Escola.

\section{Bibliografía}

Aschero, C. A. (1975). Ensayo para una clasificación morfológica de artefactos líticos aplicada a estudios tipológicos comparativos. Informe presentado a CONICET. Ms.

Aschero, C. A. (1983). Ensayo para una clasificación morfológica de artefactos líticos. Apéndice A y B. Cátedra de Ergología y Tecnología. Facultad de Filosofía y Letras. Universidad de Buenos Aires, Argentina. Ms.

Aschero, C.A. Y Hocsman S. (2004). Revisando cuestiones tipológicas en torno a la clasificación de artefactos bifaciales. En: Temas de Arqueología. Análisis Lítico. M. Ramos, A. Acosta y D. Loponte (Ed.), 7-25. Universidad 
Nacional de Luján. Luján, Argentina.

Bull, W. (1997). Discontinuos ephemeral streams. Geomorfphology 19, 227-276.

Cabrera, A.L. (1976). Regiones fitogeográficas argentinas. En: W.F. Kugler (Ed.) Enciclopedia argentina de agricultura y jardinería. Tomo 2. $2^{\circ}$ edición. Acme. Buenos Aires, Argentina. Fascículo 1, 1-85.

Candia Halupczok, D. (2015). Geomorfología del piedemonte Nororiental de la sierra de Las Quijadas. Provincia de San Luis. (Tesis de grado). Universidad Nacional de San Luis, San Luis, Argentina.

Franco, N. V. (1991). El aprovisionamiento de los recursos líticos en el Area Interserrana Bonaerense. Shincal 3 (2): 39-51.

Heider G., Gogichaishvili A., Gogorza C., Irurzun A., Ojeda G, Chiesa J., Greco C. (Abril de 2018). Estudios geoarqueológicos en el sector semi-árido occidental de la provincia de San Luis. En C. A. Baied (Coordinador), VII Congreso Nacional de Arqueometría. Materialidad, Arqueología y Patrimonio. Eje Temático 13. San Miguel de Tucumán, Tucumán, Argentina.
Nelson, M. C. 1991. The study of technological organization. Archaeological Method and Theory. M. B. Schiffer (Ed.), Vol. 3: 57-100. The University of Arizona Press. Tucson.

Ojeda G., Candia Hazlupck D., Chiesa J.O., Heider G., Gogichaishvili A., Greco C. (Septiembre de 2018). Geomorfología del arroyo Los Hornos y su vinculación con registros arqueológicos. Piedemonte Oriental Sierra de Las Quijadas. San Luis. Argentina. En O.L. Córdoba (Presidencia), Séptimo Congreso Argentino de Cuaternario y Geomorfología. Puerto Madryn, Argentina

Outes, F. F. (1926). Algunos datos sobre la arqueología de la provincia de San Luis. Physis 8, 275-304.

Renfrew C. y Bahn P. (1998). Archaeology. Theories, methods and practice. Thames and Hudson, Londres.

Vignati M. A. (1938). El "horno de tierra" y el significados de las "tinajas" de las provincias del occidente argentino. Physis, 9, 241-253.

Winchkler, G. (2006). Diccionario de uso para la descripción de objetos líticos. Recuperado en http:/www. winchkler.com.ar 